\title{
SUPPLEMENTO
}

\section{Congresso Nazionale sull'Aferesi Terapeutica}

Amici,

accogliervi a Bari in occasione dell'VIII Congresso Nazionale di Aferesi Terapeutica proposto dal Gruppo di Studio della Società Italiana di Nefrologia è stato un onore e un piacere.

Possiamo senz'altro dire che oggi, grazie al tumultuoso sviluppo tecnologico, l'aferesi terapeutica è diventata un'arma sempre più precisa ed efficace nella rimozione dei tossici che vogliamo sottrarre.

E possiamo aggiungere, senza timore di venire smentiti, che oggi non esiste disciplina della scienza medica dove l'aferesi terapeutica non abbia trovato possibilità di impiego, rivestendo, al tempo stesso, il doppio ruolo di metodica di applicazione clinica e di ricerca scientifica.

Eppure, non si è fatto abbastanza per darle il ruolo che merita. Sarebbe auspicabile riacquistare la nostra creatività di Medici dediti alla battaglia contro la malattia, evitando le gabbie dei numeri e delle statistiche e verificando, con la nostra capacità di indagine, i processi patogenetici responsabili, al fine di attribuire all'aferesi terapeutica i suoi cardini di efficacia e le sue prerogative di sicurezza.

Nell'impegnativo ed entusiasmante compito che ci attende, abbiamo unito le nostre forze con quelle degli amici Trasfusionisti; abbiamo trasmesso a molte discipline mediche le potenzialità e i meriti dell'aferesi terapeutica e il Congresso che si è svolto a Bari è stato il risultato di questo sforzo comune, mirando a essere non solo la continuazione di quanto già fatto, ma il terreno coltivato da cui siamo certi di cogliere già i primi frutti.

Gli argomenti proposti dal programma, che, accanto alla tradizione, ha inserito temi di rinnovata ispirazione, sono stati mirabilmente svolti dai Relatori presenti e il loro sforzo è stato tradotto in articoli che fanno parte di questo numero speciale dedicato all'Aferesi Terapeutica.

Da qui ci auguriamo si tragga lo spunto per uno sviluppo ulteriore, denso di soddisfazioni e risultati.

A Bari, ci abbiamo sempre creduto e ci crediamo ancora ed è a Bari che abbiamo capito di essere ormai una grande famiglia. 
